\title{
Promoção da alimentação saudável na infância: fragilidades no contexto da Atenção Básica
}

\author{
Promoting a healthy diet in childhood: weaknesses \\ in the context of Primary Health Care
}

\author{
Ariadne Barbosa do Nascimento Einloft \\ Rosângela Minardi Mitre Cotta ${ }^{1}$ \\ Raquel Maria Amaral Araújo ${ }^{1}$
}

${ }^{1}$ Programa de PósGraduação em Ciência da Nutrição, Departamento de Nutrição e Saúde, Centro de Ciências Biológicas e da Saúde, Universidade Federal de Viçosa. Campus Universitário s/n. 36570000 Viçosa MG Brasil. ariadneeinloft@gmail.com

\begin{abstract}
This paper reports the experience of the incorporation of perceptions of social actors in the investigation of the weaknesses of the process of implementation of programs that aim to promote a healthy diet in childhood. It involves qualitative research carried out in a medium-sized Brazilian municipality in which the participants were nurses and community health agents. The data were collected through semi-structured questionnaires as well as focus groups. The findings indicated that the quality and continuity of actions to promote a healthy diet were limited due to the precarious working conditions, hence the strong demand for adequate orientation due to the unfavorable context where child health is concerned. Although the reality of peripheral countries points to an incipience in program evaluation, the incorporation of qualitative investigation broadened by the perspective of the participants may constitute an important tool of participation and social responsibility. This minimizes political effects and verticalized and discontinuous actions, helping the generation of opportune and adequate information for the understanding of the different local contexts and social experiences.
\end{abstract}

Key words Qualitative research, Child nutrition, Health promotion
Resumo O trabalho versa sobre a experiência da incorporação das percepções de atores sociais na investigação das fragilidades do processo de implementação de programas de promoção da alimentação saudável na infância. Trata-se de uma pesquisa qualitativa, realizada em um município brasileiro de médio porte, onde os participantes foram profissionais enfermeiros e agentes comunitários de saúde. Os dados foram coletados por meio de questionários semiestruturados e grupos focais. Os achados incluíram precarização das condições de trabalho em um contexto desfavorável à saúde infantil, onde persiste forte demanda por atendimento qualificado. Embora na realidade de países periféricos a utilização de metodologias qualitativas seja dificultada pela incipiência em avaliação de programas, sua utilização, ampliada pela perspectiva dos participantes, pode se configurar em um importante instrumento de participação e responsabilização social, minimizando efeitos de politicas e ações verticalizadas e descontínuas, auxiliando na geração de informações oportunas e adequadas ao entendimento dos diferentes contextos locais e experiências de atores sociais.

Palavras-chave Pesquisa qualitativa, Nutrição da criança, Promoção da saúde 


\section{Introdução}

Nas últimas três décadas, o Brasil passou por sucessivas mudanças socioeconômicas, de urbanização, de atenção médica e na saúde da população ${ }^{1}$, o que se refletiu de forma significativa na saúde infantil. Embora tal fato tenha permitido a superação da meta de redução da mortalidade infantil do Objetivo do Milênio número $4^{2-4}$, esta não se deu de maneira uniforme, persistindo diferenças importantes entre regiões geográficas, classes sociais e etnias, fruto das distorções da estrutura social e da concentração de renda vivenciadas no país ${ }^{3-5}$.

Reconhecendo o impacto da alimentação na morbimortalidade infantil, organizações internacionais ${ }^{4,6}$ enfatizam há mais de uma década a necessidade de políticas de promoção da alimentação saudável para lactentes e crianças de primeira infância, baseadas principalmente na proteção e apoio à amamentação exclusiva e à alimentação complementar adequada e oportuna.

No Brasil, a Política Nacional de Alimentação e Nutrição reposicionou a questão alimentar e nutricional na agenda das políticas públicas do setor saúde ${ }^{7}$, enfatizando a importância de práticas alimentares e estilos de vida saudáveis como um componente importante para a promoção da saúde já nos primeiros anos de vida ${ }^{8}$. Nesta perspectiva surgiram diversas iniciativas, como a Rede Amamenta Brasil ${ }^{9}$, a Estratégia Nacional para Alimentação Complementar Saudável $(\text { ENPACS })^{10} \mathrm{e}$ a Estratégia Amamenta e Alimenta Brasil ${ }^{11}$, como resposta para um déficit das políticas nacionais de alimentação infantil (notadamente o apoio ao aleitamento materno), cujo foco tradicionalmente esteve na perspectiva hospitalar ou no apoio legal ${ }^{10}$.

Contudo, a despeito do reconhecido papel de intervenções nutricionais na saúde infantil e dos esforços despendidos para a implantação de estratégias de alimentação e nutrição, sua implementação tem mostrado importantes limitações ${ }^{12}$. Percebe-se que o país passa por avanços e retrocessos, de acordo com as diretrizes de governo assumidas pelos gestores responsáveis, especialmente na área de alimentação e nutrição, cujos programas são historicamente marcados pela descontinuidade ${ }^{13}$ e subnotificação de dados em sistemas de informação ${ }^{14}$.

Buscando responder à demanda crescente pela avaliação de programas e reconhecendo a realidade nacional brasileira de subnotificação e limitação de dados em sistemas de informação oficiais, foi objetivo deste estudo investigar as fra- gilidades do processo de implementação de programas de promoção da alimentação saudável na infância, na perspectiva dos profissionais da Atenção Básica. Assumindo também a impossibilidade de separação dos fenômenos sociais de seus contextos ${ }^{15}$, utilizou-se a pesquisa qualitativa como meio de dar voz a atores significantes, mas usualmente silenciados, visando auxiliar o direcionamento de estratégias de saúde.

\section{Métodos}

\section{Tipo de estudo}

Trata-se de uma pesquisa qualitativa, realizada entre outubro de 2012 a dezembro de 2013, no município de Viçosa, Zona da Mata do Estado de Minas Gerais, construída dentro da perspectiva da Avaliação Centrada nos Participantes para o aprofundamento do entendimento de fatores como restrições operacionais, características individuais de membros da equipe, além das perspectivas e preocupações dos atores envolvidos ${ }^{16}$.

\section{Participantes}

O critério para seleção do município foi o de capacitação de profissionais de todas as unidades de saúde da Atenção Básica local em estratégias de alimentação infantil.

Participaram do estudo profissionais de saúde capacitados em estratégias de alimentação infantil que tivessem contato próximo com este grupo populacional, seja por meio de consultas, grupos operativos ou visitas domiciliares, entendendo a capacitação no contexto da Política Nacional de Educação Permanente em Saúde ${ }^{17}$. Foram excluídos profissionais que, embora atendessem crianças ou integrassem atividades que englobassem alimentação infantil, não houvessem sido capacitados nesta temática. Desta forma, participaram do estudo enfermeiros coordenadores da Atenção Básica/gestores de unidades de Estratégia de Saúde da Família (ESF) e agentes comunitários de saúde (ACS).

\section{Instrumento de coleta de dados}

Para cada grupo de atores (enfermeiros e agentes comunitários) foi elaborado um roteiro de entrevista específico e semiestruturado, com questões fechadas para caracterização e abertas para apreensão das percepções e experiências dos entrevistados, abordando questões relacionadas à 
implementação de ações e estratégias de promoção da alimentação infantil.

Em virtude do interesse na discussão dos aspectos profissionais, valorativos e experienciais das ações de alimentação e cuidado da saúde infantil, optou-se também pela realização de grupos focais com os profissionais de saúde em função da sua característica de permitir a livre expressão dos participantes, em caráter qualitativo e com maior aprofundamento dos temas abordados ${ }^{18}$. Para tal, foi elaborado um conjunto de perguntas diretivas, idênticas para ambos os grupos.

\section{Procedimentos}

A coleta de dados se deu em dois momentos. No primeiro, foram entrevistados na totalidade 79 agentes comunitários de saúde em seus locais de trabalho.

No segundo momento, quando foram realizados os grupos focais, foram convidados todos os enfermeiros coordenadores das unidades de ESF (15), além de um ACS representante de cada unidade, selecionado por sorteio. Deste momento, realizado nas dependências da Secretaria de Saúde local, participaram nove enfermeiros e sete agentes comunitários de saúde. Foram realizados encontros distintos para profissionais de nível médio (agentes) e superior (enfermeiros), para minimizar possíveis inibições decorrentes de questões hierárquicas e diferenças profissionais.

\section{Instrumento de análise de dados}

Os dados quantitativos foram tabulados no Excel 6.0. Os dados qualitativos foram analisados pelo método de Análise de Conteúdo ${ }^{19,20}$.

\section{Questões éticas}

O projeto de pesquisa foi submetido ao Comitê de Ética de Pesquisas com Seres Humanos, da Universidade Federal de Viçosa, respeitando os aspectos éticos, conforme Resolução 466/12 Conselho Nacional de Saúde.

\section{Resultados}

A análise do perfil dos profissionais de saúde (Tabela 1) apontou como característica marcante e comum entre os grupos o frágil vínculo empre- gatício, com a quase totalidade dos profissionais vinculados à administração local sob regime de contrato de trabalho temporário.

Como ensino médio completo é a formação exigida para ingresso na função de agente comunitário de saúde e que entre estes apenas 24,05\% referiram formação específica na área de saúde, optou-se por investigar algumas questões sobre a capacitação dos mesmos na promoção da alimentação saudável após seu ingresso na ESF (Tabela 2), tendo sido possível perceber algumas limitações nesta área.

Conquanto $74,68 \%$ tenha recebido algum tipo de capacitação, 46,84\% referiram a realização desta há mais de 1 ano e avaliaram negativamente as que foram realizadas $(84,81 \%)$. A importância deste tipo de atividade para a prática cotidiana de atenção à criança foi reconhecida pelo grupo $(72,15 \%)$, mas a metodologia utilizada não conseguiu contemplar todas as expectativas e necessidades destes profissionais.

Em relação às ações desenvolvidas visando a promoção da saúde infantil, os ACS $(\mathrm{N}=79)$ relataram que a maioria das esquipes de $\operatorname{ESF}(78,48 \%)$ realizava atividades de acompanhamento de rotina de crianças menores de 2 anos, normalmente consultas de puericultura; porém, para 36,71\% das equipes não havia definição clara da periodicidade de realização deste tipo de atividade. Normalmente, as ações desenvolvidas se restringiam a aferições antropométricas $(45,57 \%)$ ou associação de aferições antropométricas e orientações gerais sobre alimentação, cuidados e vacinação (39,24\%). Visitas domiciliares também foram citadas como complementares às ações desenvolvidas nas unidades (3,80\%). Dos ACS entrevistados, 7,59\% não souberam descrever as ações desenvolvidas, e 3,80\% relataram não realizar nenhum tipo de ação voltada para a saúde infantil em suas unidades.

A análise das perspectivas e preocupações dos ACS e enfermeiros apresentadas nos grupos focais mostrou que, ainda que exista o reconhecimento de uma realidade que demande ações de promoção da alimentação saudável durante a infância, ao mesmo tempo a qualidade e continuidade destas são limitadas por questões profissionais e organizacionais dos serviços (Figura 1).

Das três dimensões que emergiram dos grupos focais, a Profissional e a Organizacional foram as mais exploradas pelos profissionais $\mathrm{e}$ mostram grande relação com a viabilidade de implementação de programas de promoção da alimentação saudável na infância (Quadro 1). 
Tabela 1. Perfil dos agentes comunitários de saúde e de enfermeiros componentes de equipes de Estratégia Saúde da Família. Viçosa, MG, Brasil (2012-2013).

\begin{tabular}{|c|c|c|c|c|}
\hline \multirow[t]{2}{*}{ Característica dos entrevistados } & \multicolumn{2}{|c|}{$\begin{array}{c}\text { Agentes comunitário } \\
\text { de saúde }(\mathrm{N}=79)\end{array}$} & \multicolumn{2}{|c|}{$\begin{array}{c}\text { Enfermeiros Profissionais } \\
\text { capacitados }(\mathrm{N}=15)\end{array}$} \\
\hline & $\mathbf{N}$ & $\%$ & $\mathbf{N}$ & $\%$ \\
\hline \multicolumn{5}{|l|}{ Sexo } \\
\hline Feminino & 76 & 96,20 & 13 & 86,67 \\
\hline Masculino & 3 & 3,80 & 2 & 13,33 \\
\hline \multicolumn{5}{|l|}{ Escolaridade } \\
\hline Ensino médio incompleto & 1 & 1,27 & 0 & 0 \\
\hline Ensino médio completo & 62 & 78,48 & 0 & 0 \\
\hline Superior incompleto & 9 & 11,39 & 0 & 0 \\
\hline Superior completo & 7 & 8,86 & 15 & 100,00 \\
\hline Pós-graduação & 0 & 0 & 5 & 33,33 \\
\hline \multicolumn{5}{|c|}{ Curso técnico ou especialização na área da saúde * } \\
\hline Sim & 19 & 24,05 & & \\
\hline Não & 60 & 75,95 & & \\
\hline \multicolumn{5}{|l|}{ Vinculação } \\
\hline Efetivo & 0 & 0,00 & 0 & 0 \\
\hline Não-efetivo & 75 & 94,94 & 15 & 100,00 \\
\hline Outros & 4 & 5,06 & 0 & 0 \\
\hline \multicolumn{5}{|l|}{ Tipo de equipe } \\
\hline Simples & 46 & 58,20 & 12 & 80,00 \\
\hline Ampliada & 33 & 41,80 & 3 & 20,00 \\
\hline Outro tipo de serviço & 79 & 100,00 & 0 & 0 \\
\hline \multicolumn{5}{|l|}{ Tempo de trabalho na ESF } \\
\hline Meses a 2 anos & 35 & 44,30 & 0 & 0 \\
\hline 3 a 9 anos & 41 & 51,90 & 15 & 100,00 \\
\hline$>$ de 10 anos & 3 & 3,80 & 0 & 0 \\
\hline
\end{tabular}

* Questão específica para agentes comunitários de saúde

Tabela 2. Avaliação das capacitações sobre promoção da alimentação saudável focalizadas em crianças menores de dois anos pela perspectiva dos Agentes Comunitários de Saúde. Viçosa, MG, Brasil (2012-2013).

\begin{tabular}{|c|c|c|c|}
\hline Aspectos avaliados & Resposta & $\mathbf{N}$ & $\%$ \\
\hline \multirow{2}{*}{$\begin{array}{l}\text { Capacitação em promoção da alimentação } \\
\text { saudável para crianças menores de } 2 \text { anos }(\mathrm{N}=79)\end{array}$} & Sim & 59 & 74,68 \\
\hline & Não & 20 & 25,32 \\
\hline \multirow{4}{*}{$\begin{array}{l}\text { Tempo decorrido desde a última capacitação } \\
(\mathrm{N}=79)\end{array}$} & Menos de 1 ano & 16 & 20,25 \\
\hline & 1 a 4 anos & 37 & 46,84 \\
\hline & Mais de 4 anos & 6 & 7,59 \\
\hline & Não foi capacitado & 20 & 25,32 \\
\hline \multirow{3}{*}{$\begin{array}{l}\text { Utilização prática do conhecimento adquirido na } \\
\text { capacitação }(\mathrm{N}=79)\end{array}$} & $\operatorname{Sim}$ & 57 & 72,15 \\
\hline & Não & 3 & 3,80 \\
\hline & Não foi capacitado & 19 & 24,05 \\
\hline \multirow[t]{8}{*}{ Avaliação da capacitação (N=79) } & Avaliação positiva & 12 & 15,19 \\
\hline & Avaliação negativa $(84,81 \%)$ & 67 & \\
\hline & Continuidade & 6 & 7,59 \\
\hline & Frequência & 6 & 7,59 \\
\hline & Abordagem dinâmica e prática & 2 & 2,53 \\
\hline & Duração & 5 & 6,33 \\
\hline & Formato & 15 & 18,99 \\
\hline & Não descreveu & 33 & 41,77 \\
\hline \multirow{6}{*}{$\begin{array}{l}\text { Profissional de referência dos ACS’s para questões } \\
\text { de alimentação e nutrição infantil após realização } \\
\text { das capacitações }(\mathrm{N}=79)\end{array}$} & Enfermeiro apenas & 29 & 36,71 \\
\hline & Médico apenas & 2 & 2,53 \\
\hline & Nutricionista apenas & 5 & 6,33 \\
\hline & Membros da equipe ${ }^{\star}$ & 33 & 41,77 \\
\hline & Outros (livros, internet) & 4 & 5,06 \\
\hline & Não citou & 6 & 7,60 \\
\hline
\end{tabular}




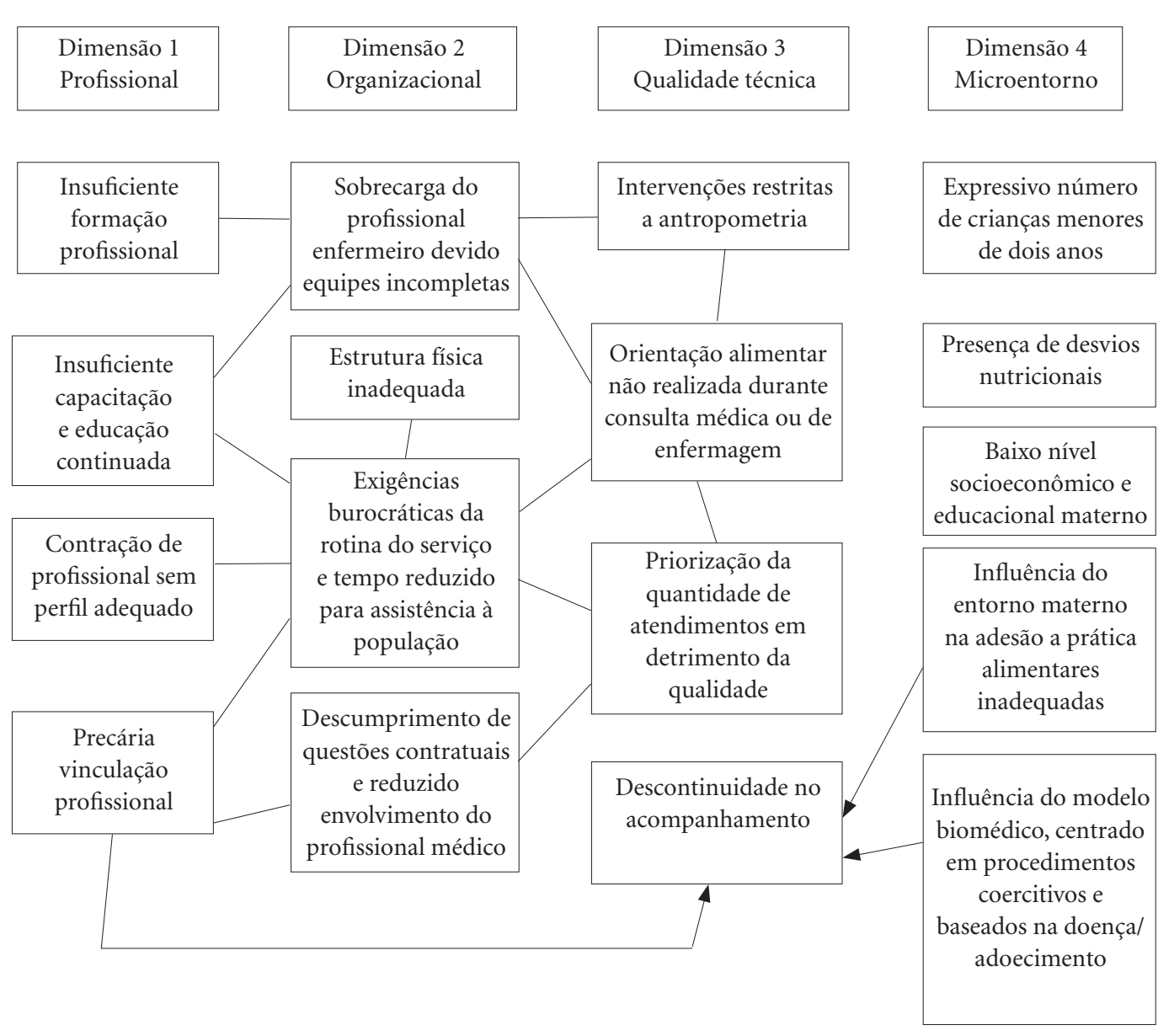

Figura 1. Sumarização das dimensões, subdimensões e possíveis interrelações emergentes das percepções apresentadas por enfermeiros e agentes comunitários de saúde sobre ações de promoção da alimentação saudável infantil em unidades de Estratégia de Saúde da Família. Viçosa, MG, Brasil (2013).

\section{Discussão}

Os serviços oferecidos por equipes de Atenção Básica, incluindo programas baseados em comunidades como a Estratégia de Saúde da Família, são considerados um lócus ideal para a realização de intervenções nutricionais; sua eficácia, porém, depende de um sistema de prestação de cuidados de saúde que funcione suficientemente bem em termos de qualidade e cobertura ${ }^{21}$. A despeito disso, ainda que iniciativas de programas de alimentação infantil tenham se mostrado eficazes na redução de desvios nutricionais, raramente são encontrados estudos que descrevam seu desenho, implementação ou sustentabilidade ${ }^{22}$. Entre os encontrados, a maioria descreve cenários de países periféricos, com limitações comuns, ainda que distantes geograficamente da realidade brasileira $^{23-26}$.

\section{Condições de trabalho - Frágil vínculo/Rotatividade}

A rotatividade profissional causada pelo frágil vínculo empregatício foi um dos principais limitantes da continuidade e qualidade das ações de promoção da saúde infantil listados pelos participantes deste estudo. Em adição também foram apontados o funcionamento de equipes incompletas, descumprimento de questões contratuais (especialmente no que se refere ao cumprimento de carga horária de trabalho do profissional médico) e contratação de profissionais sem perfil adequado para atuação na ESF. A relação entre 
Quadro 1. Sumarização das dimensões, subdimensões e depoimentos dos enfermeiros e agentes comunitários de saúde sobre ações de promoção da alimentação saudável infantil em unidades de Estratégia de Saúde da Família. Viçosa, MG, Brasil (2013).

\begin{tabular}{|c|c|c|}
\hline Dimensão & Subdimensão & Depoimento \\
\hline \multirow[t]{4}{*}{ Profissional } & - Formação profissional & $\begin{array}{l}\text { ACS2: Não, eu tive treinamento assim, entre nós ali dentro } \\
\text { [agentes], aquelas mais velhas me treinaram ali, no jeito que elas } \\
\text { sabem, a fazer o trabalho. }\end{array}$ \\
\hline & - Educação continuada & $\begin{array}{l}\text { ENF3: Até teve um treinamento aqui [secretaria] para os } \\
\text { agentes, mas assim, fica muito a desejar. [...] Tem que ser na } \\
\text { prática, a realidade. Os cursos são muito "papelzinho", sabe, } \\
\text { aquela coisa linda... } \\
\text { ACS3: [...] tinha muito slide, tinha muita coisa para você } \\
\text { assistir, uma coisa assim. E muita leitura, né? [...] Agora quanto } \\
\text { você não esta ali participando, você está só ouvindo, acaba que } \\
\text { a mente cansa e você acaba não dando nem atenção nem para } \\
\text { aquilo que tá sendo falado. }\end{array}$ \\
\hline & - Perfil profissional & $\begin{array}{l}\text { ENF1: O médico para participar é raro [de intervenções } \\
\text { nutricionais]. Tem que ser um especialista em medicina da } \\
\text { família. }\end{array}$ \\
\hline & - Vinculação profissional & $\begin{array}{l}\text { ENF9: [sobre a falta de vinculação efetiva] alguns profissionais } \\
\text { com quem eu trabalhei foram treinados, aliás, a maioria, foram } \\
\text { treinados, o que demandou tempo, dinheiro, local, o tempo } \\
\text { do profissional. Foram treinados e capacitados e depois de um } \\
\text { tempo foi todo mundo embora. } \\
\text { ACS3: [...] A pessoa não começa a confiar em você não é no } \\
\text { primeiro dia, é ao longo do tempo [..] Agora aí vem outra } \\
\text { pessoa para a mesma área [devido rotatividade profissional], aí } \\
\text { até aquela pessoa pegar confiança leva um bom tempo. Aí vai } \\
\text { trocando, o pessoal vai desgastando também [...] }\end{array}$ \\
\hline \multirow[t]{5}{*}{ Organizacional } & $\begin{array}{l}\text { - Demandas profissionais } \\
\text { e divisão do trabalho }\end{array}$ & $\begin{array}{l}\text { ENF 9: A maioria dos médicos fica pouco tempo nas unidades, } \\
\text { então a gente tá pegando no pé deles para eles ficarem as oito } \\
\text { horas. } \\
\text { ENF9: Então muitas vezes a gente não tem o tempo que a gente } \\
\text { gostaria. [...] seria ótimo se a gente pudesse estar sensibilizando } \\
\text { as pessoas Mas a gente não tem esse tempo, entendeu? } \\
\text { Simplesmente me mandam: ô, vocês vão ter que fazer tem que } \\
\text { atualizar até setembro e pronto! }\end{array}$ \\
\hline & - Estrutura física & $\begin{array}{l}\text { ENF1: Estrutura física para receber os profissionais? Aí vai } \\
\text { depender. Quem tem uma unidade moderna tem [estrutura]. } \\
\text { Mas a realidade da maioria é casa alugada, sem adaptação, sem } \\
\text { estrutura nenhuma de serviço de saúde. }\end{array}$ \\
\hline & - Exigências burocráticas & $\begin{array}{l}\text { ENF3: A gente não tem tempo de atender o paciente, acolher } \\
\text { o paciente [devido demandas burocráticas]! Aí mandar uma } \\
\text { apostila: "o acolhimento do paciente"... Como você vai acolher } \\
\text { o paciente, se você tem uma mesa lotada de papel, de tudo... } \\
\text { Nosso Deus! Ai a gente fica meio... eu fico revoltada com isso! }\end{array}$ \\
\hline & - Questões contratuais & $\begin{array}{l}\text { ENF2: [...] Por exemplo, eu sei que eu não tenho vínculo ali, que } \\
\text { eu posso ter meu contrato rescindido a qualquer momento, [..] } \\
\text { Aí você fala, nossa gente, eu já não tenho segurança nenhuma, } \\
\text { eu já tô trabalhando desse jeito tumultuado, com um monte de } \\
\text { serviço, tudo isso mexe com a cabeça da gente! }\end{array}$ \\
\hline & $\begin{array}{l}\text { - Envolvimento } \\
\text { profissional }\end{array}$ & $\begin{array}{l}\text { ENF5: [..] isso vai começar dar efeito [profissional médico } \\
\text { cumprir a carga horária] porque agora é que a gente vai ter } \\
\text { tempo de sentar com o médico, porque ele sabe que não pode ir } \\
\text { embora. [...] vai ser uma coisa que às vezes ele não vai engolir, } \\
\text { mas vai ter que fazer. }\end{array}$ \\
\hline
\end{tabular}


Quadro 1. continuação

\begin{tabular}{|c|c|c|}
\hline Dimensão & Subdimensão & Depoimento \\
\hline \multirow[t]{4}{*}{$\begin{array}{l}\text { Qualidade } \\
\text { técnica }\end{array}$} & - Tipo de intervenção & $\begin{array}{l}\text { ENF9: Na maioria dos PSF's não está acontecendo puericultura, } \\
\text { acontece uma pesagem das criança [...] e a mãe vai embora, sem } \\
\text { nenhuma informação. }\end{array}$ \\
\hline & $\begin{array}{l}\text { - Realização de } \\
\text { orientação alimentar }\end{array}$ & $\begin{array}{l}\text { ENF4: Eu até o período de amamentação, até os seis meses de } \\
\text { amamentação exclusiva eu ainda acho tranquilo. Daí pra frente, } \\
\text { não sei fazer! }\end{array}$ \\
\hline & $\begin{array}{l}\text { - Qualidade das } \\
\text { intervenções X } \\
\text { quantidade das } \\
\text { intervenções }\end{array}$ & $\begin{array}{l}\text { ENF1: [...] mais vale o médico que atende } 40 \text { numa manhã, } \\
\text { do que um médico que atende, por exemplo, doze, mas dentro } \\
\text { do que é preconizado [qualidade]. Então, hoje vale mais um } \\
\text { número registrado em papel, do que qualidade. } \\
\text { ENF7: E outra, uma coisa muito errada, eles querem } \\
\text { quantidade, número de atendimentos. E com os números que } \\
\text { eles querem a gente não dá conta de fazer. [realizar atividades } \\
\text { preventivas e de promoção] } \\
\text { ENF4: A gente faz para a criança sobreviver. É isso que é a } \\
\text { verdade! }\end{array}$ \\
\hline & $\begin{array}{l}\text { - Continuidade das } \\
\text { intervenções }\end{array}$ & $\begin{array}{l}\text { ACS7: Nunca teve [puericultura]. Por mais que a gente } \\
\text { incentiva, tudo, não tem jeito. A gente vai de porta em porta, } \\
\text { conversa, explica, elas mesmas falam [mães]: pra que, não tem } \\
\text { retorno nenhum! Para que nós vamos lá só pesar? }\end{array}$ \\
\hline \multirow[t]{5}{*}{ Microentorno } & - População infantil & $\begin{array}{l}\text { ENF7: [...] e tem muita demanda de criança. Muita demanda, } \\
\text { as mães vão na puericultura, sabe, as crianças vão. São crianças } \\
\text { necessitadas, tem desnutrição, tem sobrepeso. }\end{array}$ \\
\hline & - Desvios nutricionais & $\begin{array}{l}\text { ENF1: Vem mudando. Só que invés de mudar para normalizar, } \\
\text { tipo assim, está trocando um problema. Da desnutrição saiu } \\
\text { para o sobrepeso e não para o normal. }\end{array}$ \\
\hline & $\begin{array}{l}\text { - Nível socioeconômico e } \\
\text { educacional materno }\end{array}$ & $\begin{array}{l}\text { ENF 1: A minha questão é de informação, assim, as vezes } \\
\text { a informação não entra na cabeça dela, porque o nível } \\
\text { sociocultural do meu bairro é muito baixo }\end{array}$ \\
\hline & - Entorno materno & $\begin{array}{l}\text { ACS7: Ela falou que foi criada assim, ela cria do mesmo jeito. } \\
\text { [...] Por exemplo, eu acabo de ter filho. Minha mãe tá comigo } \\
\text { ali. Minha mãe já passou por sete gestações, ganhou sete filhos. } \\
\text { Aí vem você, que é uma agente que não tem preparo nenhum, } \\
\text { porque a gente não tem preparo nenhum. Você vai confiar em } \\
\text { quem? Eu vou confiar nela ou em você? Eu vou confiar nela. Eu } \\
\text { vou confiar na minha mãe. }\end{array}$ \\
\hline & $\begin{array}{l}\text { - Modelos de atenção à } \\
\text { saúde }\end{array}$ & $\begin{array}{l}\text { ACS6: Tipo assim, qual é a minha palavra [valor da orientação] } \\
\text { perto da de um médico, pediatra, conhecido, famoso? } \\
\text { ACS5: Eu acho que na enfermeira, no médico, acho que eles } \\
\text { [população] acabam tendo mais confiança, "ah é o enfermeiro, } \\
\text { o médico que tá passando", né, porque a gente é comum como } \\
\text { eles, não tem estudo para isso, então não passa essa confiança } \\
\text { também. } \\
\text { ACS6: Quem tem acesso faz acompanhamento com pediatra. }\end{array}$ \\
\hline
\end{tabular}

contratos temporários e relações trabalhistas precárias resultando em descontinuidade na prestação de serviços, por dificuldade de fixação da mão de obra, foi apontada na revisão de literatu- ra realizada por Arantes et al. ${ }^{27}$, que ressaltaram a insustentabilidade do próprio modelo da ESF nestas condições. A renda anual e o bem-estar econômico proveniente de condições adequadas 
de trabalho também mostram associação positiva com a satisfação no trabalho e com a saúde geral do trabalhador de saúde ${ }^{28}$.

Ainda que os profissionais reconheçam a importância das ações de promoção e prevenção, como consultas de puericultura e orientação alimentar, a manutenção de intervenções mecanizadas reprodutoras da fragmentação do cuidado $^{29}$ também foi um achado deste estudo. A sobrecarga dos profissionais enfermeiros acarretada por equipes incompletas e questões burocráticas de gerenciamento em detrimento às questões de promoção e prevenção $0^{29,30}$, foi a principal justificativa apontada pelos entrevistados para esta situação.

A relação entre superposição de atribuições e sobrecarga de trabalho ${ }^{30}$ reforça a crítica sobre a divisão de tarefas, sobretudo o pouco envolvimento dos médicos, também observado neste estudo. Normalmente as consultas de puericultura são realizadas apenas por um profissional de saúde (na maioria dos casos pelo profissional de enfermagem), de forma isolada e específica ao seu conhecimento técnico, em um contexto onde a construção de projetos terapêuticos preconizado pela ESF se torna inviabilizada pelo trabalho em equipe onde as relações profissionais são do tipo agrupamento. Neste modelo, a predominância do modelo biomédico reforça relações assimétricas de subordinação, desvalorizando saberes e ações de outros âmbitos da produção do cuidado (tais como o educativo e o preventivo), que se configuram como periféricos ao trabalho nuclear, ou seja, à assistência médica individual ${ }^{31}$.

Embora a submissão de profissionais de saúde a condições laborais frustrantes e desgastantes tenha sido mais descrita em ambientes hospitalares $^{32,33}$, é possível fazer um paralelo com os resultados deste estudo. Descontinuidade no fornecimento de recursos materiais, inadequabilidade de equipamentos e estrutura física ${ }^{33}$ podem potencializar fatores de estresse já bastante presentes na dinâmica de trabalho das equipes em condições estáveis, seja na Atenção Básica ou em ambientes hospitalares. Ghodsi et al. ${ }^{24}$, discorrendo sobre os desafios encontrados na implementação de um programa nacional de nutrição infantil, além destes fatores, citaram a escassez de recursos humanos e financeiros, instalações precárias e desatenção à motivação dos profissionais como aspectos que afetaram negativamente a implementação do referido programa.

A insatisfação profissional, associada a ambientes de trabalho negativos, também tem sido apontada como um dos constituintes da Sín- drome de Burnout ${ }^{28,30,32,34}$, que envolve exaustão (perda ou desgaste dos recursos emocionais), despersonalização (sentimentos e atitudes negativas no trabalho) e baixa realização pessoal.

Os efeitos desta situação refletem-se diretamente no formato do acompanhamento infantil: em menos da metade este era regular e incluía outras atividades além de aferição de medidas antropométricas. Para alguns autores, tal fato geraria desmotivação e baixa adesão materna, além da manutenção da cultura da busca de serviços de saúde apenas na vigência da instalação de $\operatorname{agravos}^{29,35}$.

\section{Qualidade técnica}

Intimamente relacionada à rotatividade, a educação permanente pode ser outro limitante à qualidade da atenção, uma vez que a fragilidade do vínculo pode influenciar negativamente o desenvolvimento profissional ao limitar o acúmulo de conhecimento e habilidades que tornam mais consistentes e duradouras as ações dos profissionais de saúde ${ }^{36}$.

Este trabalho evidenciou que a educação permanente, materializada no formato de capacitações, não atendia as necessidades dos profissionais de saúde, especialmente a dos ACS, que demandavam maior aprofundamento dos temas e conteúdo prático.

Por sua característica profissional peculiar de situar-se entre os saberes técnico e popular, os ACS mostraram-se particularmente mais suscetível a este tipo de limitação, por estarem continuamente expostos a uma dupla carga de desqualificação quanto ao valor de suas orientações em relação à temática da alimentação infantil. Devido ainda à forte influência biomédica na compreensão da saúde, a comunidade muitas vezes não o considera como um ator qualificado, dado sua origem local. Também os demais membros da equipe, ao desconsiderarem suas necessidades práticas, agravam esta sensação, aumentando a insegurança técnica para o exercício da orientação alimentar ${ }^{37}$.

\section{Microentorno}

A mudança do perfil epidemiológico observada nos últimos anos, causada, entre outros fatores pela transição nutricional, impôs uma mudança de enfoque na saúde no país ${ }^{38}$, ultrapassando a simples observação da presença ou ausência de doenças para a incorporação dos seus diversos determinantes, sejam eles ambien- 
tais ou sociais ${ }^{39}$. Deste modo, deve-se também compreender a dinâmica familiar e social, além de suas relações e interação com o contexto socioeconômico, histórico, político e cultural em uma abordagem mutidimensional ${ }^{39,40}$.

Neste contexto, a educação materna e seu empoderamento, bem como o fortalecimento dos sistemas de saúde, têm sido discutidos na literatura internacional como fatores considerados imprescindíveis para projeção de intervenções mais eficazes na saúde infantil ${ }^{41-43}$, particularmente na questão da amamentação e da alimentação complementar, onde o contexto sociocultural de aquisição de alimentos e o aspecto intra-doméstico do consumo alimentar são diretamente determinantes destas práticas ${ }^{25,43}$.

Os profissionais da Atenção Básica, ao considerar a família como sujeito de suas ações, lançam mão de práticas de empoderamento, que são o cerne para a promoção da saúde e ampliação da autonomia dos indivíduos ${ }^{44}$. A humanização da atenção prestada por profissionais com discernimento para assegurar sua integralidade ${ }^{45,46}$, não só nos seus aspectos orgânicos, mas também psicoafetivos e sociais, pode auxiliar a construção de condições que favoreçam as crianças a atingirem seu potencial de crescimento e desenvolvimento e, consequentemente, melhor qualidade de vida atual e futura ${ }^{47}$.

Entretanto, devido a questões culturais, os cuidados oferecidos por profissionais da Atenção Básica (como orientações alimentares, por exemplo) muitas vezes são associados à menor qualidade, sendo o cuidado especializado supervalorizado. A atenção médico-centrada, onde a atuação de outros profissionais de saúde é percebida de forma secundária ou restrita a atividades técnicas como antropometria ${ }^{44}$ ainda são muito valorizadas no grupo responsável pelo cuidado infantil ${ }^{44,48}$. Tal fato se deve ao entendimento de que por estes profissionais estarem submetidos a uma ampla variedade de demandas da população, estariam menos aptos para as necessidades específicas da criança ${ }^{49}$. Contudo, ressalta-se que neste estudo o enfermeiro foi o profissional de referência para questões de alimentação infantil dos ACS`s (provavelmente por seu papel de gestor das unidades de saúde) e o profissional médico, o único sujeito excluído por não participar de oficinas de capacitação, especialmente nesta temática.

\section{Considerações finais}

Condições profissionais desfavoráveis, contextos de forte influência do entorno materno determinando práticas alimentares inadequadas e modelo de saúde ainda centrado na atuação médica mostraram forte potencial para a descontinuidade das ações de promoção da alimentação saudável na infância.

Em relação às condições profissionais desfavoráveis, a desprecarização dos vínculos empregatícios e a implementação de políticas de valorização profissional, como concursos públicos e plano de cargos e salários, poderiam não apenas promover a permanência destes nas equipes de saúde, mas também o seu vínculo efetivo com a comunidade e o cuidado integral. Igualmente, prover as equipes com recursos humanos em suficiência e qualidade, além de recursos materiais, favoreceria os mesmos efeitos.

A qualificação por meio de uma educação permanente em saúde, que considere as demandas profissionais e locais, também se faz necessária para a autovalorização e para a valorização proveniente de outros profissionais e população. Tal ação pode se constituir em mais um catalisador da vinculação entre equipe de saúde e comunidade, bem como auxiliar na redução do impacto da formação e cultura centrada no modelo biomédico.

$\mathrm{O}$ estudo mostrou igualmente que em um cenário de desafios e fragilidades como o vivenciado pelo Sistema Único de Saúde brasileiro, de limitações físicas, estruturais, políticas e financeiras, a avaliação de programas não pode ser negligenciada como um importante suporte do processo decisório na gestão do sistema e na prestação de serviços.

Neste sentido, a incorporação das perspectivas de diferentes atores em processos avaliativos pode constituir uma importante ferramenta para avaliação da operacionalização e da qualidade de programas, uma vez que é efetivamente através destes, em suas articulações, negociações e diferentes níveis de adesão que as ações propostas se materializam.

Ainda que na realidade prática de países periféricos a utilização de metodologias qualitativas para inclusão da experiência dos atores sociais envolvidos seja dificultada pela incipiência, esta não pode ser desconsiderada. A possibilidade de emergência de conteúdos ocultos provenientes de suas falas pode preencher lacunas abertas pela subnotificação e direcionar ações enquanto este quadro não for superado. 
Ademais, a utilização da avaliação de programas, ampliada pela perspectiva dos participantes, pode se configurar em um importante instrumento de participação e responsabilização sociais, minimizando efeitos de políticas e ações verticalizadas e descontínuas, auxiliando na geração de informações oportunas e adequadas ao entendimento dos diferentes contextos locais e experiência de atores sociais.

O estudo possui as limitações intrínsecas à abordagem metodológica qualitativa, nas quais um número reduzido de sujeitos não possibilita generalizações ou inferências de seus resultados, além disso, revela percepções apenas dos profissionais de saúde envolvidos na temática. As conclusões devem ser consideradas no contexto destas limitações. O tamanho da amostra do estudo limitou sua capacidade de investigar minuciosamente as diferenças entre as respostas dos participantes, localização geográfica ou perfil profissional.

\section{Colaboradores}

ABN Einloft participou da concepção e delineamento do estudo, coleta, análise e interpretação dos dados, redação do artigo; RMA Araújo da concepção e delineamento do estudo, análise e interpretação dos dados, revisão crítica do artigo; RMM Cotta da análise e interpretação dos dados, revisão crítica do artigo.

\section{Agradecimentos}

Este estudo recebeu o apoio da CAPES - Coordenação de Aperfeiçoamento de Pessoal de Nível Superior e da Fundação de Amparo à Pesquisa do Estado de Minas Gerais. 


\section{Referências}

1. Victora CG, Aquino EML, Leal MC, Monteiro CA, Barros FC, Szwarcwald CL. Saúde de mães e crianças no Brasil: progressos e desafios. In: Saúde no Brasil. The Lancet 2011; 377(9780):1863-1876.

2. Instituto Brasileiro de Geografia e Estatística (IBGE). Censo Demográfico 2010 [acessado 2016 Mar 11]. Disponível em: http://censo2010.ibge.gov.br/resultados

3. Brasil. Ministério da Saúde (MS). Departamento de Análise de Situação de Saúde. Saúde Brasil 2011: uma análise da situação de saúde e a vigilância da saúde da mulher. Brasília: MS; 2012.

4. Fundo das Nações Unidas para a Infância (Unicef). Agenda pela infância 2015 - 2018. Desafios e propostas. Brasília: Unicef; 2014.

5. Accioly E, Saunders C, Lacerda EMA. Nutrição em obstetrícia e pediatria. $2^{\text {a }}$ ed. Rio de Janeiro: Cultura médica; 2010.

6. Organização Mundial de Saúde (OMS), Fundo das Nações Unidas para a Infância (UNICEF); International Baby Food Action Network (IBFAN). Estratégia Global para a Alimentação de Lactentes e Crianças de Primeira Infância. 2005. [acessado 2016 Mar 02]. Disponível em: http://www.ibfan.org.br/documentos/ibfan/doc-286. pdf

7. Recine E, Vasconcellos AB. Políticas nacionais e o campo da Alimentação e Nutrição em Saúde Coletiva: cenário atual. Cien Saude Colet 2011; 16(1):73-79.

8. Brasil. Ministério da Saúde (MS). Departamento de Atenção Básica/Secretaria de Atenção à Saúde. Protocolos do Sistema de Vigilância Alimentar e Nutricional - SISVAN na assistência à saúde. Brasília: MS; 2008.

9. Brasil. Ministério da Saúde (MS). Secretaria de Atenção à Saúde. Área Técnica de Saúde da Criança e Aleitamento Materno. Rede Amamenta Brasil: os primeiros passos (2007-2010). Brasília: MS; 2011.

10. Brasil. Ministério da Saúde (MS). Departamento de Atenção Básica/Secretaria de Atenção à Saúde. Estratégia Nacional para a Alimentação Complementar Saudável. Brasília: MS; 2010.

11. Brasil. Ministério da Saúde (MS). Departamento de Atenção Básica/Secretaria de Atenção à Saúde (2013). Estratégia Nacional para Promoção do Aleitamento Materno e Alimentação Complementar Saudável no Sistema Único de Saúde. Brasília: MS; 2013.

12. Venâncio SI, Martin MCN, Sanches MTC, Almeida H, Rios GS, Frias PG. Análise de implantação da Rede Amamenta Brasil: desafios e perspectivas da promoção do aleitamento materno na atenção básica. Cad Saude Publica 2013; 29(11):2261-2274.

13. Silva SP. A trajetória histórica da segurança alimentar e nutricional na agenda política nacional. Texto para discussão. Rio de Janeiro: Instituto de Pesquisa Econômica Aplicada; 2014.

14. Ferreira CS, Cherchiglia ML, Cesar CC. O Sistema de Vigilância Alimentar e Nutricional como instrumento de monitoramento da Estratégia Nacional para Alimentação Complementar Saudável. Revista Brasileira de Saúde Materno-Infantil 2013; 13(2):167-177.

15. Ribeiro J, Souza DN, Costa AP. Investigação qualitativa na área da saúde: por quê? Cien Saude Colet 2016; 21(8):2324-2324.

16. Worthen BL, Sanders JR, Fitzpatrick JL. Avaliação de programas: concepções e práticas. São Paulo: Editora Gente; 2004.
17. Brasil. Ministério da Saúde (MS). Secretaria de Gestão do Trabalho e da Educação na Saúde. Departamento de Gestão da Educação em Saúde. Política Nacional de Educação Permanente em Saúde. Brasília: MS; 2009.

18. Cruz Neto O, Moreira MR, Sucena LFM. Grupos focais e pesquisa social qualitativa: o debate orientado como técnica de investigação. In: Anais do XIII Encontro da Associação Brasileira de Estudos Populacionais; 2002; Ouro Preto [acessado 2016 Maio 20]. Disponível em: http://www.abep.nepo.unicamp.br/docs/anais/ pdf/2002/Com_JUV_PO27_Neto_texto.pdf

19. Bardin L. Análise de conteúdo. Lisboa: Edições 70; 1977.

20. Minayo MCS. O desafio do conhecimento: pesquisa qualitativa em saúde. $12^{\mathrm{a}}$ ed. São Paulo: Hucitec, 2010.

21. 21. Lutter CK, Iannotti L, Creed-Kanashiro H, Guyon A, Daelmans B, Robert R, Haider R. Key principles to improve programmes and interventions in complementary feeding. Matern Child Nutr 2013; 9(S2):101115.

22. Fabrizio CS, van Liere M, Pelto G. Identifying determinants of effective complementary feeding behaviour change interventions in developing countries. Matern Child Nutr 2014; 10(4):575-592.

23. Dewey KG, Adu-Afarwuah S. Systematic review of the efficacy and effectiveness of complementary feeding interventions in developing countries. Maternal \& Child Nutrition 2008; 4(Supl. 1):24-85.

24. Ghodsi D, Omidvar N, Rashidian A, Raghfar H, Eini-Zinab H, Ebrahimi M. Key Informants' Perceptions on the Implementation of a National Program for Improving Nutritional Status of Children in Iran. Food and Nutrition Bulletin 2016; 38(1):78-91.

25. Rahman A, Leppard M, Rashid S, Jahan N, Nasreen HE.Community perceptions of behaviour change communication interventions of the maternal neonatal and child health programme in rural Bangladesh: an exploratory study. BMC Health Serv Res. 2016; 16(a):389.

26. Robert RC, Creed-Kanashiro HM, Villasante R, Narro MR, Penny ME. Strengthening health services to deliver nutrition education to promote complementary feeding and healthy growth of infants and young children: formative research for a successful intervention in peri-urban Trujillo, Peru. Matern Child Nutr. 2017; 13(2).

27. Arantes LJ, Shimizu HE, Merchán-Hamann E. Contribuições e desafios da Estratégia Saúde da Família na Atenção Primária à Saúde no Brasil: revisão da literatura. Cien Saude Colet 2016; 21(5):1499-1510.

28. Tarcan M, Hikmet N, Schooley B, Top M, Tarcan GY. An analysis of the relationship between burnout, socio-demographic and workplace factors and job satisfaction among emergency department health professionals. Appl Nurs Res. 2017; 34:40-47.

29. Assis WD, Collet N, Reichert APS, Sá LD. Processo de trabalho da enfermeira que atua em puericultura nas unidades de saúde da família. Rev. bras. enferm. 2011; 64(1):38-46.

30. Feliciano KVO, Kovacs MH, Sarinho SW. Superposição de atribuições e autonomia técnica entre enfermeiras da Estratégia Saúde da Família. Rev Saude Publica 2010; 44(3):520-527.

31. Peduzzi M. Equipe multiprofissional de saúde: conceito e tipologia. Rev Saude Publica 2001; 35(1):103-109. 
32. Nantsupawat A, Kunaviktikul W, Nantsupawat $\mathrm{R}$, Wichaikhum OA, Thienthong $\mathrm{H}$, Poghosyan L. Effects of nurse work environment on job dissatisfaction, burnout, intention to leave. Int Nurs Rev 2017; 64(1):91-98.

33. Poghosyan L, Norful AA, Martsolf GR. Organizational structures and outcomes of newly hired and experienced nurse practitioners in New York State. Nurs Outlook 2017; 65(5):607-614.

34. Gómez-Urquiza JL, Monsalve-Reyes CS, San LuisCostas C, Fernández-Castillo R, Aquayo-Estremera R, Cañadas-de La Fuente GA. Factores de riesgo y niveles de burnout en enfermeras de atención primaria: una revisión sistemática. Atención Primaria 2017; 49(2):7785.

35. Fernandes MC, Barros AS, Silva LMS, Nóbrega MFB, Silva MRF, Torres RAM. Análise da atuação do enfermeiro na gerência de unidades básicas de saúde. Rev Bras Enferm 2010; 63(1):11-15.

36. Pinho MAL. Sofrimento no processo de trabalho na estratégia saúde da família: um diagnóstico através da terapia comunitária [dissertação]. Rio de Janeiro: Universidade Estácio de Sá; 2010.

37. Maciazeki-Gomes RC, Souza CD, Baggio L, Wachs F. O trabalho do agente comunitário de saúde na perspectiva da educação popular em saúde: possibilidades e desafios. Cien Saude Colet 2016; 21(5):1637-1646.

38. Camilo SMB, Camilo GB, Toledo GC, Camilo Júnior RD, Toledo CC. Vigilância nutricional no Brasil: criação e implementação do Sisvan. Rev APS 2011; 14(2):224-228.

39. Del Ciampo LA, Ricco RG, Daneluzzi JC, Del Ciampo IRL, Ferraz IS, Almeida CAN. O Programa de Saúde da Família e a Puericultura. Cien Saude Colet 2006; 11(3):739-743.

40. Israel B, Schulz A, Parker E, Becker A. Review of Community-Based Research: Assessing Partnership Approaches to Improve Public Health. Annual Review of Public Health 1998; 19:173-202.

41. Carlson G, J, Kordas K, Murray-Kolb LE. Associations between women's autonomy and child nutritional status: a review of the literature. Matern Child Nutr 2015: 11:452-482.

42. Cunningham K, Ruel M, Ferguson E, Uauy R. Women's empowerment and child nutritional status in South Asia: a synthesis of the literature. Matern Child Nutr 2015; 11:1-19.
43. Shankar B, Agrawal S, Beaudreault A R, Avula L, Martorell R, Osendarp S, Prabhakaran D, Mclean MS. Dietary and nutritional change in India: implications for strategies, policies, and interventions. Ann. N.Y. Acad. Sci. 2017; 1395:49-59. [acessado 2017 Abr 08]. Disponível em: http://onlinelibrary.wiley.com/doi/10.1111/ nyas.13324/full

44. Malaquias TSM, Gaíva MAM, Higarashi IH. Percepções dos familiares de crianças sobre a consulta de puericultura na estratégia saúde da família. Rev Gaúcha Enferm 2015; 36(1):62-68.

45. Brasil. Ministério da Saúde (MS). Parecer CNE/CES 1133/2001. Diretrizes Curriculares Nacionais dos Cursos de Graduação em Enfermagem, Medicina e Nutrição. Diário Oficial da União 2001; 3 out.

46. Coriolano-Marinus MWL, Andrade RS, Ruiz-Moreno LL, Lídia e Lima LS. Comunicação entre trabalhadores de saúde e usuários no cuidado à criança menor de dois anos no contexto de uma unidade de saúde da família. Interface (Botucatu) 2015; 19(53):311-324.

47. Einloft ABN, Araújo RMA, Cotta RMM. Pesquisa qualitativa em avaliação de programas de saúde: emergência de conteúdos e vozes ocultos pela ausência de dados oficiais e subnotificação. In: Atas do V Congresso Íbero-Americano em Investigação Qualitativa; 2016 Porto, Portugal. v. 2. p.1111-1120.

48. Campos RMC, Ribeiro CA, Silva CV, Saparolli ECL. Consulta de enfermagem em puericultura: a vivência do enfermeiro na Estratégia de Saúde da Família. Rev. esc. enferm. USP 2011; 45(3):566-574.

49. Reichert APS, Rodrigues PF, Albuquerque TM, Collet N, Minayo MCS. Vínculo entre enfermeiros e mães de crianças menores de dois anos: percepção de enfermeiros. Cien Saude Colet 2016; 21(8):2375-2382.

Artigo apresentado em 30/01/2017

Aprovado em 25/07/2017

Versão final apresentada em 15/08/2017 